Spin Physics (SPIN2014)

International Journal of Modern Physics: Conference Series

Vol. 40 (2016) 1660096 (7 pages)

(C) The Author(s)

DOI: $10.1142 / \mathrm{S} 201019451660096 \mathrm{X}$

\title{
NICA Facility in Polarized Proton and Deuteron Mode
}

\author{
A. D. Kovalenko*, A. V. Butenko, V. D. Kekelidze and V. A. Mikhaylov \\ Joint Institute for Nuclear Research, 6, Joliot-Curie str. Dubna, \\ 141980 Moscow Region, Russia \\ *kovalen@dubna.ru \\ A. M. Kondratenko and M. A. Kondratenko \\ Scientific and Technique Laboratory "Zaryad", 6/1 Lavrentiev Prospect., \\ Novosibirsk, 630090, Russia \\ Yu. N. Filatov \\ Moscow Institute of Physics and Technology, Dolgoprudny, Moscow Region, Russia
}

Published 29 February 2016

\begin{abstract}
NICA project at JINR is aimed at the experiments with polarized protons and deuterons at both as fixed target and colliding mode over beam momentum range from 2 to $13.5 \mathrm{GeV} / \mathrm{c}$. Polarized beams are injected into collider from the Nuclotronsuperconducting synchrotron. Dynamic solenoid "Siberian snakes" are proposed to prevent resonance depolarization of proton beam during acceleration in the Nuclotron up to momentum of $6 \mathrm{GeV} / \mathrm{c}$ and further in the collider up to the maximum momentum after storage and stochastic cooling of necessary number of particles in each ring. By means of pair of the Snakes placed in the opposite collider straight sections "spin transparency" mode is provided. Stabilization and control of the polarization is reached due to "weak field" solenoids integrated in the lattice. The proposed scheme of the polarization control is universal and can be used for different ion spices $\left(p, d, t, H e^{3} \ldots\right)$.
\end{abstract}

Keywords: Polarization; collider; proton; deuteron.

PACS numbers: 29.27.Bd, 29.27.Hj, 41.45.Ak

\section{Scheme of the Complex}

The NICA complex at JINR assume two research programs, namely: high energy heavy ion physics and spin physics studies with polarized protons and deuterons [1-3]. In this paper we briefly describe the status of design the NICA technical concept in the context of polarized proton and deuteron beams. The main elements

This is an Open Access article published by World Scientific Publishing Company. It is distributed under the terms of the Creative Commons Attribution 3.0 (CC-BY) License. Further distribution of this work is permitted, provided the original work is properly cited. 


\section{Superconducting accelerator complex NICA (Nuclotron based Ion Collider fAcility)}

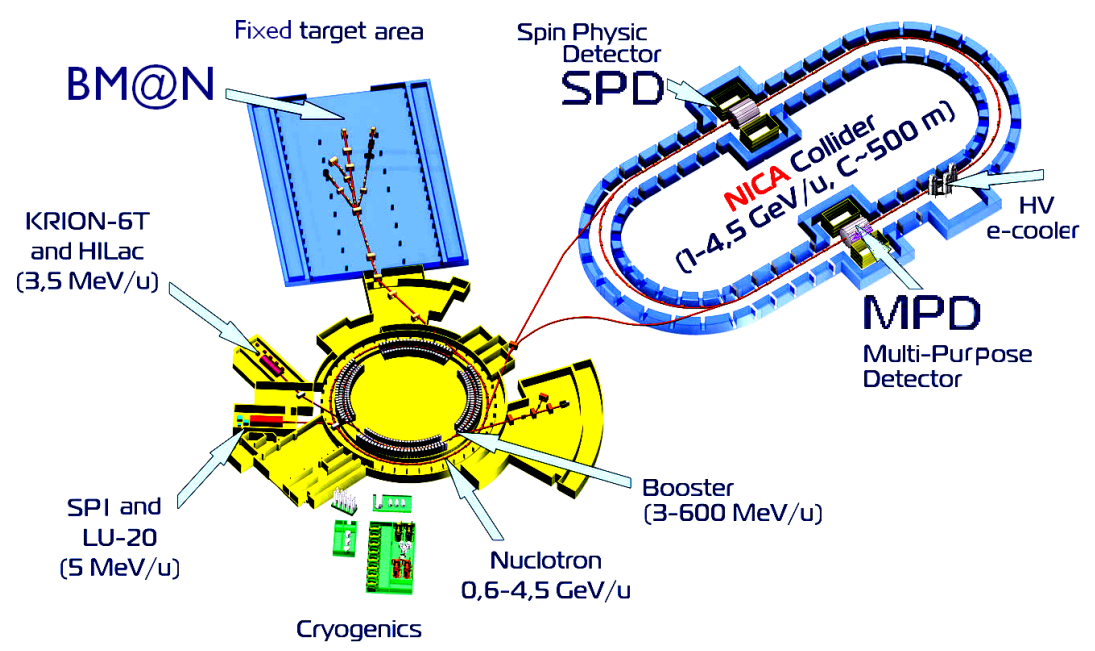

Fig. 1. NICA complex at LHEP JINR.

of NICA complex (Fig. 1) include the heavy ion source and source of polarized ions (proton and deuteron) SPI, with corresponding linacs, existing superconducting accelerator, $6 \mathrm{~A} \cdot \mathrm{GeV}$ strong-focusing synchrotron - Nuclotron, new superconducting Booster synchrotron, the collider NICA with two detectors - MPD (Multi-Purpose Detector) for heavy ion studies and SPD (Spin Physics Detector), as well as experimental hall for fixed target experiments with beams extracted from the Nuclotron. According to the NICA project, longitudinal and vertical polarization are required at SPD and MPD detectors for proton and deuteron beams. Experiments should be carried out up to the maximum energy $\sqrt{ } s_{\mathrm{NN}}$ of $27 \mathrm{GeV}$ at polarized protons and $\sqrt{ } s_{\mathrm{NN}}$ of $13 \mathrm{GeV} / \mathrm{u}$ at polarized deuterons.

\section{Polarized Ion Source}

The new polarized ion source is commissioning now. It was designed and constructed as an universal high pulsed intensity source of polarized deuterons and protons based on a charge-exchange plasma ionizer $[4,5]$. The output $\uparrow \mathrm{D}^{+}\left(\uparrow \mathrm{H}^{+}\right)$current of the source is expected to be at a level of $10 \mathrm{~mA}$. The expected polarization will reach up to $90 \%$ of the maximum vector $( \pm 1)$ for $\uparrow \mathrm{D}^{+}\left(\uparrow \mathrm{H}^{+}\right)$and tensor $(+1,-2)$ for $\uparrow \mathrm{D}^{+}$polarization. The project is designed and constructed in close cooperation with INR of RAS (Moscow). The equipment available from the CIPIOS ion source (IUCF, Bloomington, USA) is partially used for SPI. The source will deliver to the linac $10 \mu \mathrm{s}$ pulsed polarized proton or deuteron beam with intensity up to $(1-2) \cdot 10^{11}$ particles per pulse and repetition rate up to $1 \mathrm{~Hz}$. The Alvarez-type linac LU-20 used as the Nuclotron injector was designed as proton accelerator from 
$600 \mathrm{keV}$ to $20 \mathrm{MeV}$. It was modified to accelerate ions with charge-to-mass ratio $q / A>0.33$ to $5 \mathrm{MeV} / \mathrm{u}$ at $2 \beta \lambda$ mode. The pulse transformer voltage up to $700 \mathrm{kV}$ is now used to feed the accelerating tube of the LU-20 pre-injector. The new preinjector will be based on the RFQ section. The SPI is now commissioning at test bench, whereas the front-end linac section is under manufacturing [6].

\section{Polarized Protons and Deuterons Acceleration at Nuclotron}

\subsection{Polarized deuterons}

Acceleration of polarized deuterons at the Synchrophasotron was done for the first time in 1984 and at the Nuclotron in 2002 [7, 8]. There are no dangerous spin resonances at polarized deuteron acceleration up to an energy of $5.6 \mathrm{GeV} / \mathrm{u}$. Vertical polarization direction is stable at polarized deuteron acceleration in the Nuclotron, whereas in the NICA magnetic arcs the polarization is coincides with the collider median plane. Thus, it is necessary to change the polarization direction over an angle of $\pi / 2$ before the beam injection to the collider from the Nuclotron. It could be done by means of installation solenoid in the injection channel with the longitudinal field integral from 12 to $80 \mathrm{Tm}$ in the beam momentum range from 2 to $13.5 \mathrm{GeV} / \mathrm{c}$.

\subsection{Polarized protons}

To eliminate crossing of numerous dangerous depolarizing spin resonances in Nuclotron in the case of protons, solenoid Siberian snake will be installed into the second straight section of the accelerator ring [9]. The section consists of two free spaces of $3.5 \mathrm{~m}$ long each separated by structural $D$ quad that could be used for the snake. Structure of the straight section with the solenoids is shown in Fig. 2, where $\mathrm{F}$ and $\mathrm{D}$ are focusing and defocusing quadrupoles of the Nuclotron FODO structure.

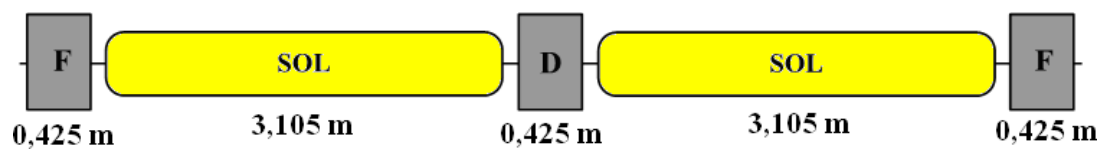

Fig. 2. Layout of the straight section with the solenoid Siberian snake.

The analysis of particle dynamics in the case of a strong coupling of betatron oscillations due to the solenoid snake has been performed. It was shown [10], stable operation of the accelerator in polarized mode can be provided without the use of additional compensating quadrupoles. Such approach makes it possible the most effective use of free space and reduce substantially the maximum of the solenoid field amplitude. The use of a full Siberian snake, rotating the spin over $\pi$ radians, makes it possible to save polarization of protons at any arbitrary values of the betatron tunes. It is possible to reduce the field integral using a partial snake; nevertheless the limitation to the operating tunes will be occurred. For a half snake, 
Table 1. The main parameters of the snake solenoid at beam momentum of $6 \mathrm{GeV} / \mathrm{c}$.

\begin{tabular}{lcc}
\hline \multicolumn{1}{c}{ Snake Solenoid parameters } & Half Snake & Full Snake \\
\hline Cable outer diameter (with insulation), mm & 9 & 9 \\
Number of turns per meter & 111 & 111 \\
Solenoid inner diameter, mm & 100 & 100 \\
Number of layers & 2 & 2 \\
Maximum Magnetic field, T & 1.7 & 3.4 \\
Field ramp, T/s & $2-3$ & $2-3$ \\
Maximum supply current, kA & 6 & 12 \\
Stored energy per section, kJ & 69.6 & 278 \\
\hline
\end{tabular}

rotating spin over an angle of $\pi / 2$ the field integral is reduced by a factor of 2 , whereas stable area of the tunes $\nu_{x, y}$ is limited to $\cos 2 \pi \nu_{x, y}>0$. The snake solenoid field integral should be changed proportionally to the particle momentum during the acceleration. Superconducting pulsed solenoid with the field $\operatorname{ramp} d B / d t \approx 2-$ $3 \mathrm{~T} / \mathrm{s}$ can be manufactured based on the Nuclotron-type hollow high-current NbTi composite superconducting cable cooled with two-phase helium flow [11]. The main necessary parameters of the solenoid are shown in Table 1. Take note, full snake designed for the particle momentum of $6 \mathrm{GeV} / \mathrm{c}$ can be operate as half snake for the total range of the Nuclotron magnetic rigidity. Thus, it is possible to optimize the beam injection energy in the collider to reach the luminosity.

The possibility is also opened to provide experiments with polarized protons at fixed target over the total energy range. Similar to the deuteron case, it is necessary to provide proper direction of the polarization vector (within the collider median plane). The same solenoids in the beam transportation line to collider can be used for that purpose. In the case of a full snake polarization is always seats within the collider median plane.

\section{Collider NICA in Spin Transparency Mode}

To control efficiently polarization of protons and deuterons as well it was proposed to use the collider in, so called, "spin transparency" mode realized if two identical solenoid Siberian Snakes are introduced in the opposite straight sections of the collider (Fig. 3) [3].

The Snakes are separated symmetrically by MPD and SPD detectors. Spin transparency mode means that the influence of the fields created by the two Snakes and the collider's arcs doesn't change the spin direction from turn to turn, i.e. the magnetic system is transparent for the spin. The NICA collider with two Snakes becomes similar to JLAB figure-8 shaped collider [12].

The unique feature of a spin transparency accelerator is the possibility to obtain any particle polarization using small magnetic field integrals [13]. The proton and deuteron polarization in the NICA collider ring can be efficiently controlled by "weak" solenoids. Any angle between the polarization and the beam direction lying 


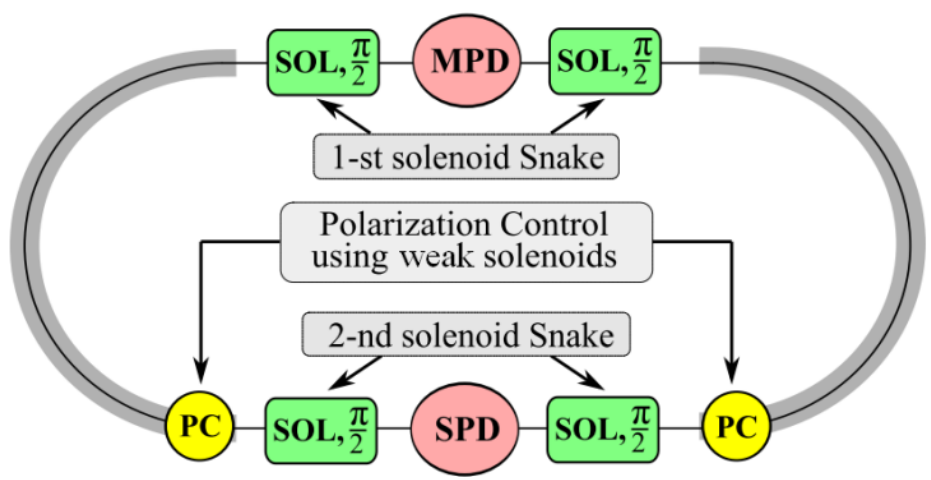

Fig. 3. The polarization control scheme of protons and deuterons in NICA collider.

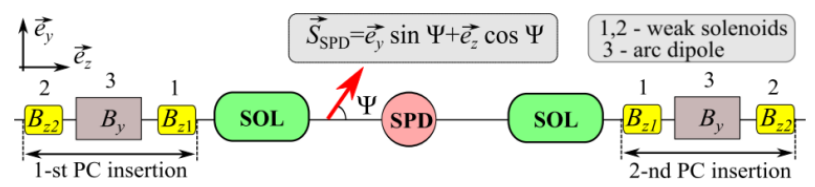

Fig. 4. Polarization control by means of weak solenoids in NICA collider.

in the vertical plane of SPD detector can be obtained by introducing polarization control (PC) insertions. Each PC insertion is based on two weak solenoids separated by arc's dipoles. A symmetric scheme of polarization control with two PC insertions located at the both sides of the SPD detector is presented in Fig. 4.

The required spin rotation angles of the longitudinal fields in each PC insertion are given for small values of the spin tune $\nu$ by

$$
\phi_{z 1}=\pi \nu \frac{\sin \left(\phi_{y}-\Psi\right)}{\sin \phi_{y}}, \quad \phi_{z 2}=\pi \nu \frac{\sin \Psi}{\sin \phi_{y}},
$$

where $\phi_{z 1}=(1+G)\left(B_{z} L\right)_{1} / B \rho$ and $\phi_{z 2}=(1+G)\left(B_{z} L\right)_{2} / B \rho$ are the spin rotation angles of the solenoids, $\varphi_{y}=\gamma G \alpha$ is the spin rotation angle of the dipole section between the solenoids, $\alpha$ is the orbit bending angle between the solenoids, $\Psi$ is the angle between the polarization and the beam direction, and $B \rho$ is the magnetic rigidity.

The work spin tune $\nu$ induced by PC insertions should exceed substantially the strength of zero spin resonance. The strength is determined by the beam emittances and by misalignments and manufacturing imperfections of the collider's magnetic system. These values are estimated to $\nu_{p}=0.01$ and $\nu_{d}=0.001$ for proton and deuteron case respectively. Schematic placement of the strong solenoids for Siberian snakes and weak solenoids for polarization control is shown in Fig. 5.

Optimal orbital angle between the PC insertion solenoids is $4.5^{\circ}$ (one regular dipole) and $90^{\circ}$ (quarter of the arc) for proton and deuteron case respectively. The main parameters of the strong and weak solenoids are presented in Table 2. 


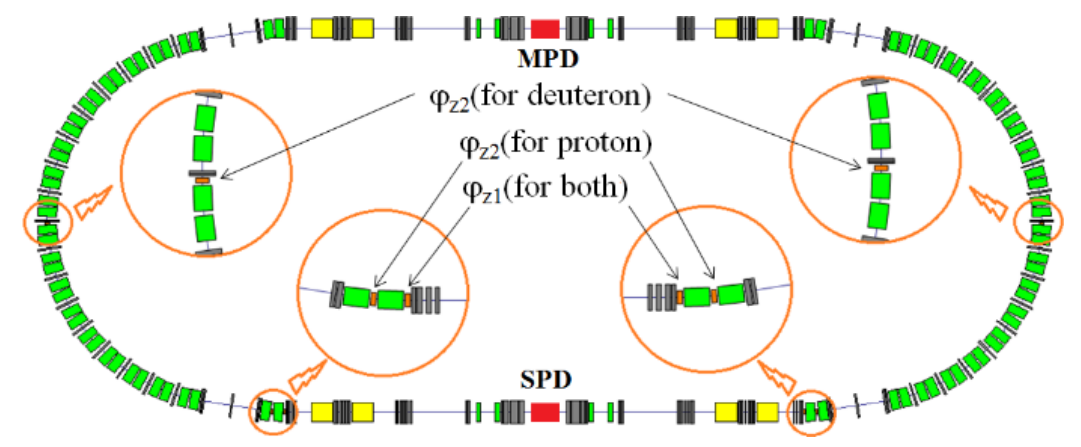

Fig. 5. Strong (marked with yellow) and weak (increased icons) solenoids in NICA collider.

Table 2. The main parameters of the solenoids in NICA collider.

\begin{tabular}{ccccc}
\hline Type of solenoids & number & $B_{\max }, \mathrm{T}$ & $L, \mathrm{~m}$ & $B L, \mathrm{~T} \cdot \mathrm{m}$ \\
\hline Strong solenoid for Siberian snakes: & & & & \\
$\begin{array}{c}\text { proton/deuteron case } \\
\text { Weak solenoid for polarization control }\end{array}$ & 8 & $2.3 / 7.3$ & 5.5 & $0 \div 12.5 / 40$ \\
\hline
\end{tabular}

The Snakes field integral should be changed proportional to the particle momentum during the beam acceleration. The field integral of weak solenoids are changed in accordance with the Eq. (1) and don't exceed $0.6 \mathrm{~T} \cdot \mathrm{m}$. Magnetic field of weak solenoids can be ramped up and down enough rapidly (much less than a second) that can allow the use of PC insertions for spin-flipping. The presented scheme makes it possible to control the polarization in the both SPD and MPD detectors. The angles between the polarization and velocity of the particle in the detectors are connected as: $\Psi_{\mathrm{MPD}}=\gamma G \pi+\Psi_{\mathrm{SPD}}$. The important feature of the considered scheme is the excluding of resonance depolarization usually due to crossing of numerous spin resonances. In the proposed scheme the spin tune is kept unchanged during the acceleration and at the spin gymnastic during the physics data taken.

\section{Conclusion}

Polarization control system in the NICA complex makes it possible:

- to provide polarization control of different particles (p, d, ${ }^{3} \mathrm{He}, \ldots$ );

- to provide any direction of polarization in the vertical plane SPD and MPD detectors;

- to solve the problems of spin matching at injection in the NICA collider and polarization measurement as well;

- to eliminate resonance depolarization during acceleration;

- to realize Spin Flipping System;

- to control polarization in SPD and MPD detectors without any change of beam orbital characteristics. 


\section{References}

1. V. D. Kekelidze et al., Status of NICA project at JINR, PoS 085, pp. 1-9, SISSA, Italy (2012).

2. A. D. Kovalenko et al., The NICA Facility in Polarized Proton Operation Mode, IPAC'11, TUPZ004 (2011).

3. A. D. Kovalenko et al., Polarized Deuterons and Protons at NICA@JINR, Pepan, Vol. 45, No. 1, pp. 262-264 (2014).

4. V. Fimushkin et al., EPJ ST, 162, pp. 275-280 (2008).

5. V. Fimushkin et al., Pepan, Vol. 45, No. 1, pp. 297-300 (2014).

6. A. V.Butenko et al., Development of the NICA Injection Facility, IPAC'14, WEPRO067 (2014).

7. N. G. Anishchenko et al., Journ. de Phys. Colloquia C2, Vol. 46, C2-703 (1985).

8. I. B.Issinsky et al., In: Proceedings of III Int. Symposium Deuteron-95, 1996, pp. 169175 .

9. M. A. Kondratenko et al., Orbital Parameters of Proton Beam in Nuclotron with Solenoid Siberian Snake, Proc. of XV Workshop DSPIN'2013, pp. 371-374, Dubna (2013).

10. Yu. N. Filatov et al., Solenoid Siberian Snake Without Compensation of Betatron Oscillation Coupling in Nuclotron, IPAC'14, TUPRO057 (2014).

11. A. D. Kovalenko et al., Superconducting Magnets for the NICA Facility at JINR: Status of the Design and Construction Plans, IPAC'10, MOPEB040, pp. 361-363 (2010).

12. arXiv: $1209.0757 \mathrm{v} 2$ [physics.acc-ph] (2012).

13. Yu. N. Filatov et al., Proton and Deuteron Polarization Control in NICA Collider Using Small Solenoids, Proc. of XV Workshop DSPIN'2013, pp. 351-354, Dubna (2013). 